\title{
Should my patient with inflammatory bowel disease on immunosuppressive therapy be vaccinated against influenza virus?
}

\author{
Neeraj Narula MD ${ }^{1}$, Deborah LR Yamamura MD FRCPC ${ }^{2}$, John K Marshall MD MSc FRCPC AGAF
}

\begin{abstract}
N Narula, DLR Yamamura, JK Marshall. Should my patient with inflammatory bowel disease on immunosuppressive therapy be vaccinated against influenza virus? Can J Gastroenterol 2010;24(2):121-125.
\end{abstract}

Crohn's disease and ulcerative colitis are variants of inflammatory bowel disease (IBD) for which immunosuppressive therapy is often required. Immunosuppressed patients are at increased risk for infections, including vaccine-preventable diseases such as influenza. Although several guidelines recommend routine influenza immunization for such patients, recent literature suggests that this patient population may be inadequately immunized. Current research suggests that inactivated influenza vaccines are effective, well tolerated and can be administered safely in most IBD patients. Studies in other immunosuppressed populations have also demonstrated the safety of inactivated vaccines. The present article reviews the literature regarding the safety and efficacy of influenza vaccination in IBD patients receiving immunosuppressive therapy.

Key Words: Crohn's disease; Immunization; Inflammatory bowel disease; Influenza; Ulcerative colitis; Vaccination

Prohn's disease and ulcerative colitis are both variants of inflammatory bowel disease (IBD), and affect approximately $0.5 \%$ of Canadians (1). Disease onset can occur at any age but typically peaks in the third decade of life (1). It is generally accepted that an unknown environmental agent(s) and a dysfunctional mucosal immune system in genetically susceptible individuals leads to the development of either Crohn's disease or ulcerative colitis (2). Treatment of IBD often requires the use of immunosuppressive medications including corticosteroids, purine antimetabolites, methotrexate and newer biological agents (eg, infliximab, adalimumab, certolizumab and natalizumab). As a result of immunosuppression, patients with IBD may be at higher risk for certain infections than the general population and the outcomes of these infections may be more severe $(3,4)$.

The risk of influenza infection and its complications is considered to be higher in patients undergoing immunosuppressive therapies (5). Influenza is an acute respiratory infection from which most people recover. However, adults and children with chronic illnesses such as IBD are at greater risk for severe complications such as pneumonia and death (6). Depending on the severity of the virus in any given season, between 2000 and 8000 Canadians die of influenza and its complications annually (6). Whether immunocompromised patients are more susceptible to a novel virus such as the pH1N1 (swine) strain is

\author{
Mon patient atteint d'une maladie \\ inflammatoire de l'intestin qui prend des \\ immunosuppresseurs devrait-il être vacciné \\ contre le virus de l'influenza?
}

La maladie de Crohn et la colite ulcéreuse sont des variantes des maladies inflammatoires de l'intestin (MII) souvent traitées au moyen d'immunosuppresseurs. Les patients immunosupprimés sont plus vulnérables aux infections, y compris des maladies évitables par un vaccin comme l'influenza. Même si plusieurs lignes directrices recommandent la vaccination systématique contre l'influenza chez ces patients, de récentes publications indiquent que cette population ne serait pas suffisamment vaccinée. Selon les recherches à jour, les vaccins contre l'influenza inactivé sont efficaces, bien tolérés et peuvent être administrés en toute sécurité à la plupart des patients atteints d'une MII. Des études auprès d'autres populations immunosupprimées démontrent également l'innocuité des vaccins inactivés. Le présent article contient une analyse des publications au sujet de l'innocuité et de l'efficacité du vaccin contre l'influenza chez les patients atteints d'une MII sous immunosuppresseurs.

${ }^{1}$ Department of Medicine (Division of Gastroenterology) and Farncombe Family Digestive Health Research Institute; ${ }^{2}$ Department of Pathology and Molecular Medicine, McMaster University, Hamilton, Ontario

Correspondence: Dr John K Marshall, Division of Gastroenterology (2F59), McMaster University Medical Centre, 1200 Main Street West,

Hamilton, Ontario L8N 325. Telephone 905-521-2100 ext 76782, fax 905-523-6048, e-mail marshllj@mcmaster.ca

Received for publication November 10, 2009. Accepted December 1, 2009 
trials conducted in children and healthy adults demonstrate $70 \%$ to $90 \%$ efficacy in preventing influenza infection (14). The inactivated influenza vaccine may be less effective in patients receiving immunosuppressive medications $(15,16)$. Although there are limited data on the efficacy of influenza vaccination in IBD patients, several guidelines recommend the vaccination of patients with chronic disease, including those with IBD who are immunosuppressed $(5,8-10)$. The present article reviews the current literature pertaining to the efficacy and safety of seasonal influenza vaccinations in IBD patients receiving immunosuppressive therapy.

\section{Literature search}

\section{METHODS}

A systematic search was conducted to retrieve high-quality, peer-reviewed studies of influenza vaccination in IBD. The PubMed, Medline and EMBASE databases were searched with text ("vaccination", "influenza", "colitis", "Crohn" and "inflammatory bowel disease") and MeSH terms ("Crohn's disease", "ulcerative colitis", "inflammatory bowel diseases", "influenza vaccination" and "immunization"). Results were limited to English language publications.

\section{DISCUSSION}

\section{Vaccination use in patients with IBD}

Melmed et al (17) surveyed 169 IBD patients to assess their immunization histories and exposures to known risk factors for influenza, Streptococcus pneumoniae, viral hepatitis and varicella. The majority of these patients (86\%) reported current or previous use of immunosuppressive medications. Only 41 patients (24\%) reported receiving regular influenza vaccinations. The most common reasons for nonimmunization with influenza vaccines were lack of awareness (49\%) and concern for side effects (18\%).

The findings of the Melmed et al study were consistent with reports of influenza vaccination in other at-risk populations. Sowden and Mitchell (18) found that only 53\% of patients on immunosuppressants who were attending outpatient rheumatology clinics in the United Kingdom had received influenza vaccinations. Bridges et al (19) reported that only 56\% of patients receiving methotrexate for rheumatoid arthritis (RA) were immunized against influenza. The most commonly cited reason for nonimmunization was 'never being offered the vaccine'. These data suggest that patients and health care providers are not aware of national immunization guidelines or have residual concerns about the risk-benefit trade-off in special patient populations.

\section{Efficacy of vaccination in IBD}

To date, few studies have assessed the immune response to influenza vaccine in patients with IBD (Table 1). Mamula et al (15) assessed 51 pediatric IBD patients and 29 healthy controls undergoing single-dose inactivated influenza vaccination. The authors used hemagglutinin inhibition (HI) titres of $1: 40$ or higher as a measure of the vaccine efficacy, which is considered to have better correlation with influenza protection than other methods (20). Overall, the immune response to the $\mathrm{B} /$ Hong Kong vaccine antigen was reduced (62\% versus $89 \% ; \mathrm{P}=0.0125$ ) (15). Among patients receiving infliximab and immunomodulatory therapy, responses to two of the three influenza vaccine antigens were reduced (63\% versus $95 \%$ for strain $\mathrm{A} / \mathrm{New}$ Caledonia/20/99, $\mathrm{P}=0.018$; and $33 \%$ versus $89 \%$ for strain $\mathrm{B} /$ Hong Kong/330/2001; $\mathrm{P}=0.002$ ). However, no other significant differences were observed and the vaccine was well tolerated by the test group. Only 15 patients (19\%) reported adverse events, all of which were mild, including soreness at the injection site (14\%), having a cold (5\%), influenza-like symptoms $(4 \%)$ and headache (1\%). Furthermore, vaccination did not affect the clinical activity of IBD as measured by the Pediatric Crohn's Disease Activity Index for patients with Crohn's disease, and by the Lichtiger Colitis Activity Index for patients with ulcerative colitis. Although serological conversion in patients with IBD on concomitant infliximab and immunomodulatory therapy may be inadequate, the authors recommended routine immunization for patients with IBD because of good tolerability.

During the 2007 to 2008 influenza season, Lu et al (21) conducted a prospective cohort study of the efficacy of inactivated influenza vaccine in 137 pediatric IBD patients. They obtained serum from these patients to determine a baseline influenza titre, immunized the patients with a TIV, then remeasured titres three to nine weeks later. $\mathrm{HI}$ titres of 1:40 or greater were used to define seroprotection. They found that regardless of immunosuppressive status, more patients attained seroprotection against strains A/Solomon Islands/3/2006 (H1N1) and $\mathrm{A} /$ Wisconsin/67/2005 (H3N2) than the B/Malysia/2506/2004 strain. Overall, rates of seroprotection were not affected by the use of immunosuppressive therapy; however, a subanalysis showed that those receiving antitumour necrosis factor (TNF) therapy were less likely than those on no immunosuppression to be protected against strain B ( $14 \%$ versus $39 \% ; \mathrm{P}=0.025$ ). Responses in the IBD study cohort were similar to those in a comparator cohort of healthy controls. Similar to Mamula et al (15), the authors found the vaccine to be well tolerated, with no effect on disease activity. Lu et al (21) also recommended routine influenza vaccination for IBD patients.

Gelinck et al (22) assessed the serological responses to influenza vaccine in 112 patients receiving anti-TNF therapy, including 22 with IBD. They found no significant difference in the rates of seroprotection (HI titre greater than 1:40) among patients who were treated with anti-TNF agents and patients treated with either other immunosuppressive medications or healthy controls. However, the absolute level of titres against two influenza strains was significantly lower in the anti-TNF group. The authors noted no differences in vaccine efficacy with respect to underlying disease or anti-TNF agent. No major side effects or exacerbations of disease were reported.

While all of the studies were consistent in their definition of seroprotection as $\mathrm{HI}$ titres of greater than 1:40, humoral response is only a surrogate marker for an immunoprotective state. It would be preferable to monitor patients for the development of influenza infection after receiving a TIV.

\section{Influenza vaccination in other immunosuppressed populations}

Many transplant recipients do not receive an annual influenza vaccination, largely because of physicans' concerns about triggering an allograft rejection (23). Hypothetically, nonspecific immune activation following vaccination may lead to enhanced cellular or humoral responses against donor organs; there are anecdotal reports of acute rejection subsequent to 
TABLE 1

Prospective studies of immune response to influenza vaccination in patients with inflammatory bowel disease (IBD)

Author

(reference)

year

\begin{tabular}{lll}
\hline Mamula & Prospective cohort study with & Administration of \\
et al (15) & 51 IBD patients and & influenza \\
2007 & 29 controls & vaccine
\end{tabular}

29 controls

vaccine

Lu et al (21) Prospective cohort study with Administration of

2009137 IBD patients and influenza
76 controls vaccine

Gelinck et al Prospective cohort study with (22) 2008112 patients on anti-TNF therapy (including 22 with Administration of influenza IBD) and 18 controls

Outcome(s) measured

Primary: HI before and 4 weeks after

vaccination. Seroprotection was $\mathrm{HI} \geq 1: 40$

at 4 weeks.

Secondary: Safety and clinical disease activity (measured by CDAI or LCAI)

Seroprotection (defined as $\mathrm{HI} \geq 1: 40$ to each influenza strain measured 3 to 9 weeks postvaccination), absolute antibody titres and adverse events

$\mathrm{HI}$ before and 4 weeks after vaccination, seroprotection defined as $\mathrm{HI} \geq 1: 40$

vaccine

Result

IBD patients had lower seroconversion to 1 of

3 strains. Patients on both infliximab and immunomodulators were less likely to convert 2 of 3 strains than healthy controls. Vaccine was safe and well tolerated

Most IBD patients attained seroprotection regardless of immunosuppressive therapy (especially to A strains). Patients on anti-TNF therapy were less likely to be protected against the B strain. Vaccine was safe and well tolerated No significant difference in proportion of individuals attaining a protective titre $(\mathrm{HI} \geq 1: 40)$ but absolute titre levels lower in patients on anti-TNF therapy. Vaccine was safe and well tolerated

CDAI Crohn's Disease Activity Index; HI Hemagglutinin inhibition titre; LCAI Lichtiger Colitis Activity Index; TNF Tumour necrosis factor

immunization (19). However, most studies have failed to demonstrate a definitive association between influenza vaccination and graft rejection (24-29). Burbach et al (30) and Lawal et al (31) observed no increase in rejection or liver enzyme elevation after influenza vaccination among 62 and 51 liver transplant recipients, respectively. Candon et al (23) found no increased risk of allograft rejection, as measured by antihuman leukocyte antigen antibody serum levels within 30 days of vaccination among 66 stable renal transplant recipients. Kimball et al (32) reported no excess rejection, no increase in alloantibodies and no changes in lymphocyte subpopulations among 29 vaccinated heart transplant recipients. In the largest study to date, White-Williams et al (33) also found no increase in rejection among 3601 heart transplant recipients who received an influenza vaccination. This study revealed that transplant institutions enforced varying intervals between transplantation and vaccination, but that this interval did not appear to affect rates of rejection.

Similar concerns about increased immune activity have tempered the enthusiasm for vaccination in patients with autoimmune disease (34). A review by Elkayam (35) identified several small trials in which influenza vaccination induced an adequate humoral response without inducing a clinical exacerbation of RA; however, their cumulative sample size was too small to draw any definitive conclusions.

More recently, Oren et al (36) compared the safety and immunogenicity of influenza vaccine in 14 RA patients on rituximab with 29 patients on other nonbiological diseasemodifying antirheumatic drugs. No significant worsening of any clinical or laboratory parameter of disease activity was noted in either group of patients, but those on rituximab mounted lower humoral responses ( $21 \%$ versus $67 \% ; \mathrm{P}=0.006)$. Salemi et al (37) assessed the development of seroprotective titres against influenza over three consecutive influenza seasons by administering influenza vaccination to healthy control subjects and 28 patients with stable RA who were undergoing anti-TNF treatment. Their study revealed that the RA patients did not develop clinical or biochemical disease reactivation with vaccination but were less likely to develop seroprotection against the influenza B strain. Kaine et al (38) assessed humoral responses to influenza and pneumococcal vaccination in a double-blind trial comparing adalimumab $(n=99)$ with placebo $(n=109)$ in patients with RA. In subjects without protective antibody titres at baseline, antibody responses were similar in the adalimumab and placebo groups (73.3\% and 73.9\%, respectively). Rates of disease flare, adverse events and treatment discontinuation were also similar. Overall, evidence supports the use of influenza vaccine in RA patients who are on immunosuppressive medication.

\section{Serious adverse reactions to influenza vaccine}

The most common adverse events associated with inactivated vaccines are arm soreness and redness at the injection site (39). These are mild reactions and generally resolve within two days. Systemic symptoms such as fever, arthralgia and malaise occur much less frequently, and are more likely to occur in individuals exposed to the vaccines for the first time (12). GuillainBarré syndrome (GBS) is a rare neurological condition that was associated with the 1976 swine influenza vaccine at a rate of one per 100,000 persons vaccinated (40). Since then, some studies have estimated its incidence with seasonal influenza vaccine at one per million persons vaccinated, and others $(41,42)$ have not found an association between influenza vaccination and GBS. The Advisory Committee on Immunization Practices (5) recently stated that the potential benefits of influenza vaccination in preventing serious illness, hospitalization and death outweigh the possible risk of vaccine-associated GBS. Influenza vaccines should not be given to individuals who have had an anaphylactic reaction to a previous dose or with a known immunoglobulin E-mediated hypersensitivity to eggs that manifests as swelling of the mouth and throat, hypotension, difficulty breathing or shock (13). Patients with serious acute febrile illnesses should not be vaccinated until their symptoms have resolved (13).

Observational data have raised concerns about the reactivation of IBD with influenza vaccination, but the overall quality of these data is poor. Kwan et al (43) reported on a 39-year-old woman with ulcerative colitis who was well controlled on oral mesalazine, who experienced abdominal pain and frequent bowel movements with hematochezia three days after 
vaccination, but responded to steroid induction. Another case report (44) describes a 52-year-old woman with ulcerative colitis who had been in remission without steroids for nine months; however, within hours of receiving an influenza vaccination, the woman experienced a severe exacerbation of her disease that could not be controlled with steroids and immunosuppressive medications. She became steroid-dependent, experienced many corticosteroid-induced side effects and, finally, underwent a total colectomy within three months. The patient subsequently recalled milder reactivation following a previous influenza vaccination.

\section{CONCLUSIONS}

Patients with IBD are at risk for the same vaccine-preventable illnesses as the general population. Influenza is one of the most common vaccine-preventable illnesses, but patients with IBD often fail to receive appropriate immunizations because of poor awareness and uncertainty about vaccine safety and efficacy (17). This may have significant consequences for patients taking immunosuppressive medications because they are the most vulnerable to serious complications from infection. The limited available data suggest that these patients can achieve protective titres, albeit less often than healthy controls, with good safety. Two reports of disease reactivation are insufficient reason to withhold immunization. However, physicians should administer vaccines with caution to patients with previous adverse reactions to the vaccine. Further prospective studies are needed to clarify the benefits and risks of immunization in patients with IBD.

\section{REFERENCES}

1. Bernstein CN, Wajda A, Svenson LW, et al. The epidemiology of inflammatory bowel disease in Canada: A population-based study. Am J Gastroenterol 2006;101:1559-68.

2. Baumgart DC, Sandborn WJ. Inflammatory bowel disease: Clinical aspects and established and evolving therapies. Lancet 2007;369:1641-57.

3. Toruner M, Loftus Jr EV, Harmsen WS, et al. Risk factors for opportunistic infections in patients with inflammatory bowel disease. Gastroenterology 2008;134:929-36.

4. Hutfless SM, Weng X, Liu L, et al. Mortality by medication use among patients with inflammatory bowel disease, 1996-2003. Gastroenterology 2007;133:1779-86.

5. Fiore AE, Shay DK, Broker K, et al. Prevention and control of influenza. Recommendations of the Advisory Committee on Immunization Practices (ACIP), 2008. MMWR Recomm Rep 2008;57:1-60.

6. Public Health Agency of Canada. Influenza (June 4, 2009). $<$ http://www.phac-aspc.gc.ca/influenza/index-eng.php> (Version current at October 24, 2009).

7. Kangro HO, Chong SK, Hardiman A, et al. A prospective study of viral and mycoplasma infections in chronic inflammatory bowel disease. Gastroenterology 1990;98:549-53.

8. Harper SA, Fukuda K, Uyeki TM, et al. Prevention and control of influenza: Recommendations of the Advisory Committee on Immunization Practices (ACIP). MMWR Recomm Rep 2004;53:1-40.

9. Public Health Agency of Canada. Statement on Influenza Vaccination for the 2008-2009 season (July 15, 2008). $<$ http://www.phac-aspc.gc.ca/publicat/ccdr-rmtc/08vol34/acs-3/ index-eng.php > (Version current at October 24, 2009).

10. Sands BE, Cuffari C, Katz J, et al. Guidelines for immunizations in patients with inflammatory bowel disease. Inflamm Bowel Dis 2004:10:677-92.

11. Rahier JF, Ben-Horin S, Chowers Y, et al; on behalf of the European Crohn's and Colitis Organisation (ECCO). European evidence-based consensus on the prevention, diagnosis and management of opportunistic infections in inflammatory bowel disease. JCC 2009;3:47-91.
12. Fiore $\mathrm{AE}$, Bridges $\mathrm{CB}$, Cox NJ. Seasonal influenza vaccines. Curr Top Microbiol Immunol 2009;333:43-82.

13. Public Health Agency of Canada. Statement on Seasonal Trivalent Inactivated Influenza Vaccine (TIV) for 2009-2010 (October 29, 2009) <http://www.phac-aspc.gc.ca/publicat/ccdr-rmtc/09vol35/acsdcc-6/index-eng.php> (Version current at October 29, 2009).

14. Williams MS, Wood JM. A brief history of inactivated influenza virus vaccines. In: Options for the Control of Influenza II. Hannoun C, Kenda AP, Klenk HD, Ruben FL, eds. Amsterdam: Elsevier Science Publishers, 1993:169-71.

15. Mamula P, Markowitz JE, Piccoli DA, et al. Immune response to influenza vaccine in pediatric patients with inflammatory bowel disease. Clin Gastroenterol Hepatol 2007;5:851-6.

16. Matsuzaki A, Suminoe A, Koga Y, et al. Immune response after influenza vaccination in children with cancer. Pediatr Blood Cancer 2005;45:831-7.

17. Melmed GY, Ippoliti AF, Papadakis KA, et al. Patients with inflammatory bowel disease are at risk for vaccine-preventable illnesses. Am J Gastroenterol 2006;101:1834-40.

18. Sowden E, Mitchell WS. An audit of influenza and pneumococcal vaccination in rheumatology outpatients. BMC Musculoskelet Disor 2007;8:58

19. Bridges MJ, Coady D, Kelly CA, et al. Factors influencing uptake of influenza vaccination in patients with rheumatoid arthritis. Ann Rheum Dis 2003;62:685.

20. Beyer WE, Palache AM, de Jong JC, et al. Cold-adapted live influenza vaccine versus inactivated vaccine: Systemic vaccine reactions, local and systemic antibody response, and vaccine efficacy. A meta-analysis. Vaccine 2002;20:1340-53.

21. Lu Y, Jacobson DL, Ashworth LA, et al. Immune response to influenza vaccine in children with inflammatory bowel disease. Am J Gastroenterol 2009;104:444-53.

22. Gelinck LB, van der Bijl AE, Beyer WE, et al. The effect of antitumour necrosis factor alpha treatment on the antibody response to influenza vaccination. Ann Rheum Dis 2008;67:713-6.

23. Candon S, Thervet E, Lebon P, et al. Humoral and cellular immune responses after influenza vaccination in kidney transplant recipients. Am J Transplant 2009;9:2346-54.

24. Grekas D, Alivanis P, Kiriazopoulou V, et al. Influenza vaccination on renal transplant patients is safe and serologically effective. Int J Clin Pharmacol Ther Toxicol 1993;31:553-6.

25. Kobashigawa JA,Warner-Stevenson L, Johnson BL, et al. Influenza vaccine does not cause rejection after cardiac transplantation. Transplant Proc 1993;25:2738-9.

26. Mauch TJ, Crouch NA, Freese DK, et al. Antibody response of pediatric solid organ transplant recipients to immunization against influenza virus. J Pediatr 1995;127:957-60.

27. Briggs WA, Rozek RJ, Migdal SD, et al. Influenza vaccination in kidney transplant recipients: Cellular and humoral immune responses. Ann Intern Med 1980;92:471-7.

28. Gross PA, Lee H, Wolff JA, et al. Influenza immunization in immunosuppressed children. J Pediatr 1978;92:30-5.

29. Versluis DJ, Beyer WE, Masurel N, et al. Impairment of the immune response to influenza vaccination in renal transplant recipients by cyclosporine, but not azathioprine. Transplantation 1986;42:376-9.

30. Burbach G, Bienzle U, Stark K, et al. Influenza vaccination in liver transplant recipients. Transplantation 1999;67:753-5.

31. Lawal A, Basler C, Branch A, Gutierrez J, Schwartz M, Schiano TD. Influenza vaccination in orthotopic liver transplant recipients: Absence of post administration ALT elevation. Am J Transplant 2004;4:1805-9.

32. Kimball P, Verbeke S, Flattery M, Rhodes C, Tolman D. Influenza vaccination does not promote cellular or humoral activation among heart transplant recipients. Transplantation 2000;69:2449-51.

33. White-Williams C, Brown R, Kirklin J, et al. Improving clinical practice: Should we give influenza vaccinations to heart transplant patients? J Heart Lung Transplant 2006;25:320-3.

34. Holvast B, Huckriede A, Kallenberg CG, et al. Influenza vaccination in systemic lupus erythematosus: Safe and protective? Autoimmun Rev 2007;6:300-5.

35. Elkayam O. Safety and efficacy of vaccination against influenza in patients with rheumatoid arthritis. Clin Dev Immunol 2006;13:349-51. 
36. Oren S, Mandelboim M, Braun-Moscovici Y, et al. Vaccination against influenza in rheumatoid arthritis patients: The effect of rituximab on the humoral response. Ann Rheum Dis 2008;67:937-41.

37. Salemi S, Picchianti-Diamanti A, Germano V, et al. Influenza vaccine adminstration in rheumatoid arthritis patients under treatment with TNFalpha blockers: Safety and immunogencity. Clin Immunol 2009, Oct 20 [Epub ahead of print].

38. Kaine JL, Kivitz AJ, Birbara C, Luo AY. Immune responses following administration of influenza and pneumococcal vaccines to patients with rheumatoid arthritis receiving adalimumab. J Rheumatol 2007;34:272-9.

39. Bridges CB, Thompson WW, Meltzer MI, et al. Effectiveness and cost-benefit of influenza vaccination of healthy working adults: A randomized controlled trial. JAMA 2000;284:1655-63.

40. Schonberger LB, Bregman DJ, Sullivan-Bolyai JZ, et al. Guillain-Barre syndrome following vaccination in the National Influenza Immunization Program, United States, 1976-1977. Am J Epidemiol 1979;110:105-23.

41. Juurlink DN, Stukel TA, Kwong J, et al. Guillain-Barre syndrome after influenza vaccination in adults: A population-based study. Arch Intern Med 2006;166:2217-21.

42. Kaplan JE, Katona P, Hurtwitz ES, et al. Guillain-Barre syndrome in the United States, 1979-1980 and 1980-1981. Lack of an association with influenza vaccination. JAMA 1982;248:698-700.

43. Kwan OS, Park YS, Choi JH, et al. A case of ulcerative colitis relapsed by influenza vaccination. Korean J Gastroenterol 2007;49:327-30.

44. Fields SW, Baiocco PJ, Korelitz BI. Influenza vaccinations: Should they really be encouraged for IBD patients being treated with immunosuppressives? Inflamm Bowel Dis 2009;15:649-51. 


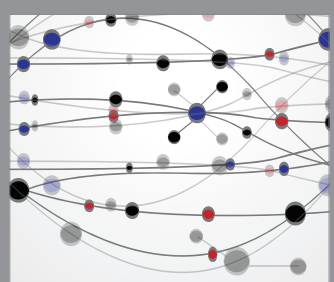

The Scientific World Journal
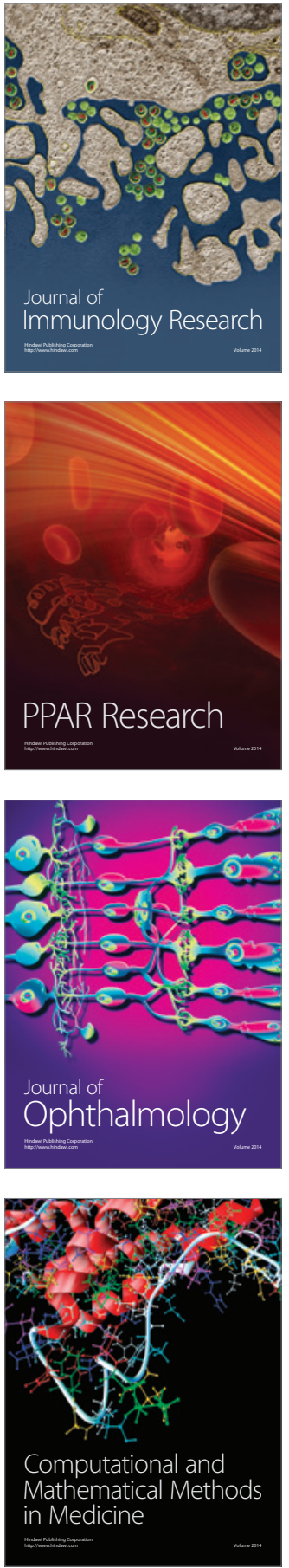

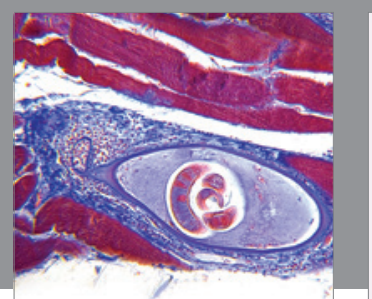

Gastroenterology Research and Practice

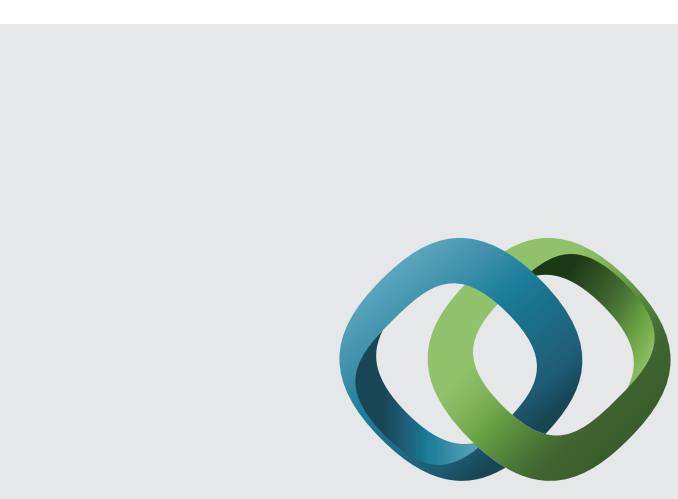

\section{Hindawi}

Submit your manuscripts at

http://www.hindawi.com
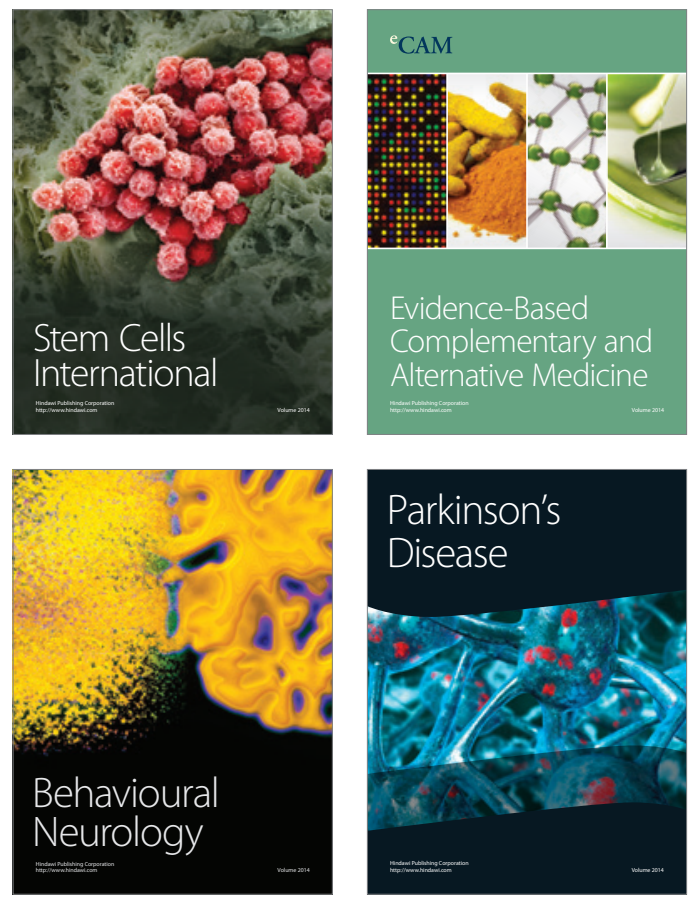
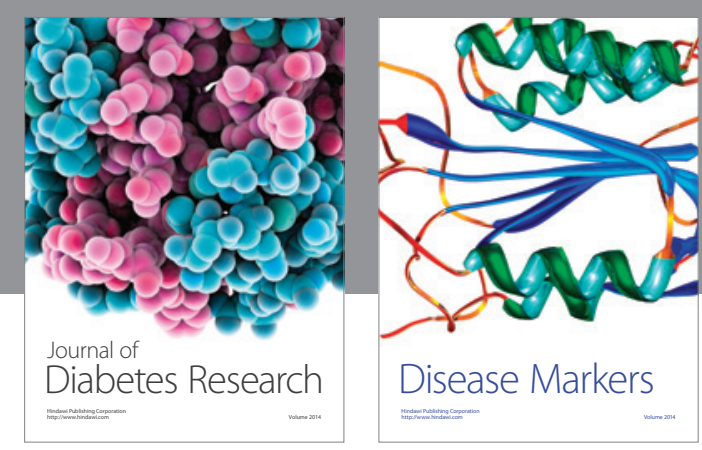

Disease Markers
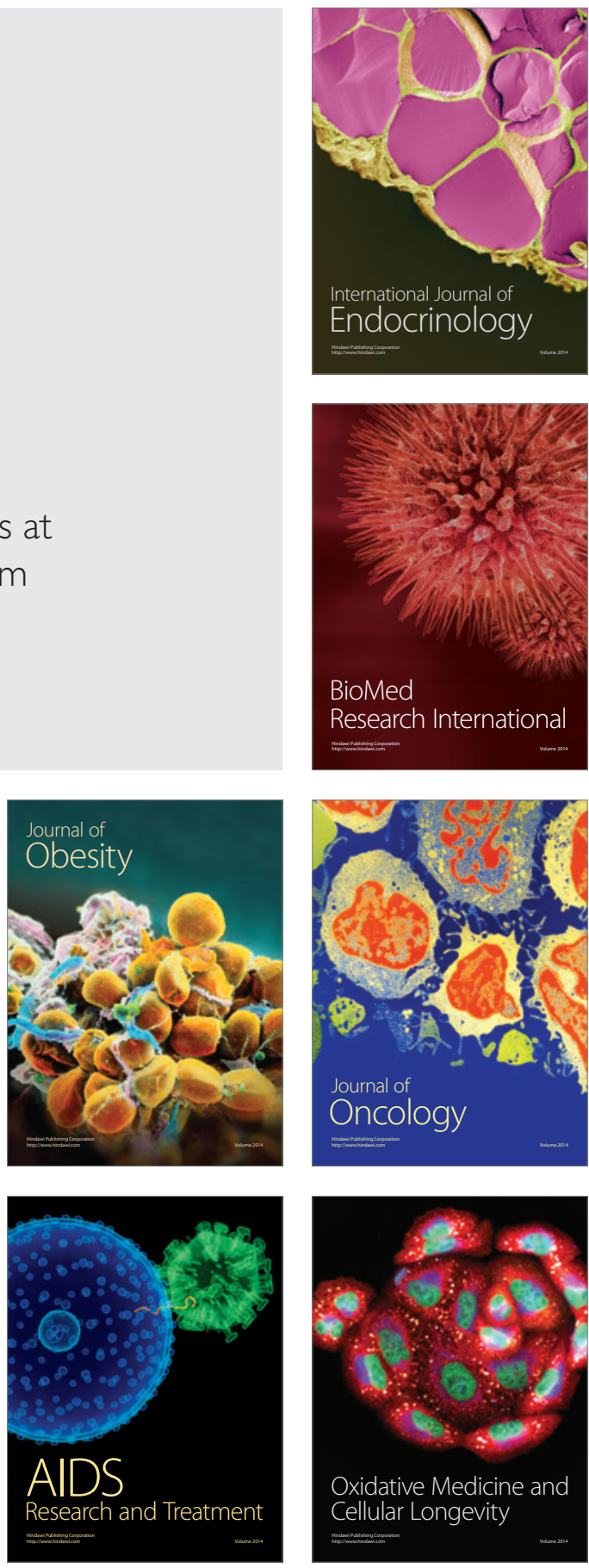